\title{
Determinants of Next Generation e-Health Network and Architecture Specifications
}

\author{
Christoph Thuemmler \\ School of Computing \\ Edinburgh Napier University \\ Edinburgh, UK \\ c.thuemmler@napier.ac.uk
}

\author{
Alois Paulin \\ Faculty of Informatics \\ Vienna University of Technology \\ Vienna, Austria \\ alois@apaulin.com
}

\author{
Ai Keow Lim \\ Celestor Ltd \\ Edinburgh, UK \\ aikeowlim@gmail.com
}

\begin{abstract}
This paper summarizes the determinants for future e-Health network and IT infrastructures in the health care environment. The paper bases on observations conducted as part of a larger study at a university hospital in Munich, and summarizes ongoing discussions, key determinants from relevant white papers, and challenges of the domain. The objective of the paper is to provide a broad overview over the implications of the e-Health domain to provide inputs in ongoing discussion on $5 \mathrm{G}$ characteristics.
\end{abstract}

Keywords - 5G; future network requirements; e-health;

\section{INTRODUCTION}

In the context of $5 \mathrm{G}$ requirements discussions next generation network and architecture specifications for the health domain have been repeatedly discussed recently. Several white papers have been compiled by different relevant organizations such as NGMN and 5G PPP [1], [2]. This was also echoed in a recent statement of the European $5 \mathrm{G}$ Association at the 2016 Mobile World Congress in Barcelona, where the socio-economic relevance of the health vertical in the context of the progressive digitalization of Europe was highlighted [3]. However, all of these publications discuss target specifications pretty much from a technological perspective in an ideal world with limited assessment of real world requirements, resulting from health economic, political and social factors. Although this might be perceived as sober and structured by some scientists this inevitably brings us back to the longstanding, controversial discussion on whether innovation is triggered by new technology or by societal change [4]

In this paper we are going to discuss most recent findings from the technical and social domain relevant to healthcare in order to shed some light on the determinants of future network specifications. We believe that trying to identify and analyze the driving forces behind future network specifications is a more accurate method to assess future requirements than assessing technical performance indicators of latest technology. In this paper we will create an outlook at future $5 \mathrm{G}$ requirements in healthcare in typical healthcare settings in Europe and elsewhere.

In order to forecast future $5 \mathrm{G}$ requirements in healthcare, we first describe in section II the current data traffic mix of an urban hospital with 1,100 beds in Munich, Germany (hereinafter referred to as the University Hospital). Section III provides an overview over emerging models and paradigms in the e-Health domain [5]. Sections IV - VIII provide thematically clustered insights into different aspects of $5 \mathrm{G}$ requirements, implications, and constraints, which are relevant to understand the requirements of future network requirements in the e-Health domain. Section IX concludes.

\section{Current Data Sources}

An investigation of the everyday use of computerized and informated systems revealed [6] that technology-supported activities in the hospital routine can be classified into four broad use categories, namely the monitoring of vital signs, the creation of digitized body scans, the storage and exchange of patient records, and communication between units. Table 1 presents the current and future estimated peak network traffic load of the University Hospital.

\section{TABle I. TABle 1. Current \& Estimated Peak Network TrafFic}

\begin{tabular}{|c|c|c|c|}
\hline & 2015 & 2025 & 2035 \\
\hline No. of beds & 1,100 & 1,050 & 1,000 \\
\hline No. of intensive care beds & 35 & 70 & 100 \\
\hline $\begin{array}{l}\text { Beds with full remote vital } \\
\text { parameter reading }\end{array}$ & - & 70 & 100 \\
\hline $\begin{array}{l}\text { Traffic stream vital parameter } \\
\text { readings }\end{array}$ & - & $56 \mathrm{Mbps}$ & $80 \mathrm{Mpbs}$ \\
\hline IoT connections /bed & - & 10 & 20 \\
\hline Traffic stream IoT & - & $9 \mathrm{Mbps}$ & $88 \mathrm{Mbps}$ \\
\hline Imagery access count (Patient/day) & 4 & 8 & 10 \\
\hline Traffic imagery access $^{\mathrm{d}}$ & 49 Mbps & $98 \mathrm{Mbps}$ & $122 \mathrm{Mbps}$ \\
\hline Live video streams surgery/room & - & 1 & 2 \\
\hline Traffic live video streams surgery ${ }^{f}$ & - & $150 \mathrm{Mbps}$ & $300 \mathrm{Mbps}$ \\
\hline $\begin{array}{l}\text { Total peak traffic (without } \\
\text { entertainment) }\end{array}$ & $\begin{array}{l}<100 \\
\text { Mbps }\end{array}$ & $\begin{array}{l}\text { ca. } 500 \\
\text { Mbps }\end{array}$ & $\begin{array}{l}\text { ca. } 600 \\
\text { Mbps }\end{array}$ \\
\hline
\end{tabular}

The data traffic mix, as described below, includes crossdomain access to patient records, patient monitoring, images and multimedia, and out-hospital data interactions.

\section{A. Cross-domain access to patient records}

The University Hospital uses the SAP system for its hospital information systems (HIS), which is a popular modular enterprise resource planning system providing a wide range of solutions to support and virtualize workflows in various business scenarios. User interacts with the frontend presentation server (client application), which in turn interacts 
with the application servers in the backend, and that subsequently interacts with the database server. Administrative (text-only) interaction with the HIS causes moderate volume of data to flow though the hospital network. An estimated of 50 users concurrently using the system (client-server interactions) would result in 25-50 kbps of network data traffic.

\section{B. Patient monitoring}

Patient's vital parameters such as blood pressure, oxygen saturation, body temperature and pulse provide valuable insight into the patient's status and is important for recognizing and responding to deteriorating patients. At the University Hospital, the blood gas analysis is the only vital parameter captured automatically in the inpatient ward and transferred directly on the patient data management system (PDMS) server whereas other vital signs assessments are written down manually on the paper file of the patient. While data generated by bio-signal monitoring devices usually amounts to less than $10 \mathrm{kbps}$ [7], [8], sensor resolution is $12 / 16 / 14$ bits with sample rates ranging from $5-50.000 \mathrm{~Hz}$, yielding in data output from 80 bps to 600 kbps respectively [9].

\section{Images and multimedia}

Images and multimedia produced and transmitted during routine operation (e.g. X-Ray, ultrasound, computer tomography (CT) and nuclear magnetic resonance (NMR) images) constitute a major portion of the hospital data traffic. The amount of data collected is about 500 GB losslesscompressed imagery per month, with a maximum of ca. 80 simultaneous connections at the University Hospital. These data are stored at the hospital's central picture archiving and communication system (PACS) in the form of digital images and communication in medicine (DICOM) files.

\section{Out-hospital data interactions}

Out-hospital stakeholders include the emergency mobile units, established doctors, research agencies, the general public, suppliers, the patient and its kin. Interactions with these stakeholders are currently unstructured and frequently improvised. Communication with the emergency mobile units such as emergency ambulance and rescue helicopter is communicated over dedicated national telecommunications systems (e.g. German BOS-Funk) [10] or the emerging TETRA (terrestrial trunked radio) systems. In spite of available technology, paper-based transfer of information is still the main mean of interactions with established doctors.

\section{EMERGING MODELS AND PARADIGMS}

\section{A. Narrowband IoT for the Integration of Smart Devices}

To assure indoor coverage of connected IoT / Smart Devices for professional health care, narrowband IoT acts as an emerging enabler to track and trace healthcare sensors, things, and smart pharmaceuticals in the hospital environment. Periodic data retrievals from sensors and other mobile equipment (e.g. m-Health systems [11]) present a relevant source of future network traffic, as well as a significant driver of measures to protect and govern thus generated Hippocratic information, as outlined further below.
To connect huge quantities of IoT-enabled devices, more than 20 years after its introduction, IPv6 is yet facing challenges regarding its realization within the IoT vision [12].

\section{B. eDelivery}

Increased reliance on electronically transmitted digital information entails the challenge to deliver the information in a way in which both partners in the exchange of data can rest assure that the transmission has succeeded. On top of that, the transmission of Hippocratic information, which by definition is of a sensible private nature, requires to keep track of which partners the information was shared with - that is, the challenge boils down to forging a bi-directionally nonrepudiable audit trail.

Bi-directionally non-repudiable audit trails in communication are well-known in legal and administrative proceedings, where communication between the involved parties relies on certified mail, which assures that the receiver must sign the receipt of a delivery. This same principle in electronic message transmission is assured by ways of eDelivery (also known as Certified e-Mail), where fair nonrepudiable communication protocols [13] automate a bidirectionally non-repudiable message transfer.

Fair non-repudiation is an emerging driver of network traffic and information exchange in e-Health systems [14], which is increasing in importance with the maturing of IoT and its application in e-Health [14].

\section{Edge Computing and Edge Clouds}

A further implication of the sensitive personal nature of Hippocratic data is the tendency to prevent such data to be transferred in the first place. To this end, edge computing [15] has emerged as a novel paradigm in which data is processed and consumed at the edge of the network, typically at the data collecting devices (such as cameras, mobile devices, sensors, etc. [15]) themselves.

Data, which care givers collect for their purposes, should be stored on private clouds, with edge clouds serving as a way to allow external stakeholders to derive results from the care giver's data without the data leaving the premises of the care provider.

\section{Network Slicing}

Telecom operators might create industry or vertical specific software defined networks (SDN) to establish encrypted endto-end communication (VPN). This means that healthcare network slices could be protected in line with specific national regulatory requirements. However, providing the much desired quality of service, which needs at least for mission critical services to be considerably better than "best effort" is not without controversy as this might interfere with the notion of net-neutrality. There is an ongoing discussion between telecom operators and the relevant regulator (BEREC) with view to certain incompatibilities between healthcare requirements in the context of for example the real time monitoring of artificial organs such as brain pacemakers, insulin-pumps and others and technologies enabling individualized medicine and the perceived need for equality with regards to net access. 


\section{E. Personalised Medicine}

Informatization [16] enables the design of medical devices, such as (artificial) organs, or drugs on the computer, so they can be tailored to fit specific patients or specific situation. To denote so-created bespoke design, the attribute "personalized" is used, which reflects in concepts such as personalized medicine, or personalized genomics.

Do-it-yourself biotechnology, organ printing, telemedicine (in terms of on-demand-provision of healthcare agent devices), etc. are then but other names used to describe the ability to manufacture healthcare agent devices of various kinds using emerging technology such as additive manufacturing (commonly known as 3D-printing).

\section{F. Robotics}

The overall impact of medical robotics on the global data traffic is expected to be rather small. However, with regards to hospital networks the amount of videos from robotic and minimal invasive procedures is expected to increase. We expect at least a volume of $100 \mathrm{~GB}$ per day to be generated by 1.000 bed hospitals with a surgical department such as the observed University Hospital.

Robotics, as used e.g. in the surgical context, is dependent on ultra-low latency, but has considerably low data bandwidth requirements with regards to the transmitted command-andcontrol messages. The fixed nature of a fully-equipped surgical theatre allows for low distances between the connected devices, which helps to keep latency low. Security and integrity of the network is paramount, as well as the reliability, while signal penetration and mobility provided to the networked devices is of low importance.

\section{G. E-Health Grids}

We are currently witnessing the emergence of national health grids such as Sundhed in Denmark [17] or ELGA in Austria [18] and other regional solutions such as SOLE in the Emilia-Romagna region Italy [19]. While these "grids" are currently functionally not much more but repositories it is foreseeable that they will technologically evolve and become centerpieces of infrastructures to deliver the transition from hospital based, expert centered systems to distributed patient centric approaches. This will strengthen the self-management capacity of patients and informal carers, make healthcare available anyhow, anywhere and at any time and increase effectiveness and efficiency of healthcare systems.

\section{RELEVANT STANDARDS FOR E-HEALTH INFRASTRUCTURE}

Some well-known standards deal with security aspects in general (ISO 27000) and health care infrastructure in particular (ISO 80001). Another important standard supports the exchange of information among medical devices (ISO 11073).

\section{A. ISO/IEC 27000 - Information security matters}

The ISO/IEC 27000 family of standards provides a structured approach to keep information assets secure. ISO/IEC 27001 for example [20], is providing the requirements for a generic information security management system, not necessarily specific to the healthcare domain, but certainly applicable in health care settings.

\section{B. ISO/IEC 80001- Application of risk management for IT- networks incorporating medical devices}

ISO/IEC 80001 looks into the safety of devices incorporated into IT-networks. It becomes in particular relevant, where "there is no single medical device manufacturer assuming responsibility for addressing the key properties of the IT-network incorporating a medical device" [21].

\section{ISO/IEEE 11073 - Health Informatics - Personal health device communications}

The 11073 family defines the components and provides specifications in order to exchange various biological parameters between different medical devices. An example would be ISO/IEEE 11073-10418:2014 which is concerned with the "normative definition of communication between personalized telehealth International Normalized Ratio (INR) devices (agents) and managers (e.g. cell phones, personal computers, personal health appliance [...] in a manner that enables plug and play interoperability" [22].

These three standards are well known to the community but despite the fact that they probably the three most popular and most important they are all but well established in the health domain. Eventually standards are well-recognized examples of best practice but they are typically not legally binding. However, bearing in mind that the examples in this paragraph do not reflect the full picture of standards relevant to medical networks there can be no doubt that the standards listed address major well recognized aspects of medical network planning, design and implementation which should be considered in deployment scenarios.

\section{INFRASTRUCTURE CHALLENGES AND LIMITATIONS OF RADIO ACCESS TECHNOLOGIES IN HEALTH CARE FACILITIES}

Typically, the majority of hospitals in Europe have either been built in the 19th or early 20th century with solid external walls or they are "new builds", typically designed as steel frame constructions. Both structural approaches are challenging to wireless communication and mobile telephony. Many hospitals have been fitted with cables to connect devices to the central IT infrastructure but most hospitals build before 2000 simply don't have a cable infrastructure with a sufficient granularity as the extend of the digitalization of our societies and in particular healthcare have been underestimated then. Many hospitals are fitted with wireless access points but coverage is typically patchy and limited to authorized users and specific data traffic. Extensions to the network are frequently considered expensive, due to required construction measures disruptive and in some cases administratively challenging, for example if the healthcare provider does not own the health facility and is dependent on the agreement of the owner. The use of Radio Access Technologies (RATs) such as $3 \mathrm{G}$ or $4 \mathrm{G}$ is dependent on physical specifications of the building hence why areas below ground or within steel frame constructions or thick walls typically lack coverage. Although modern hospitals have 
in general excellent reception in most areas the problem is the lack of reliability which is unacceptable in the health domain for example in the context of emergency calls to surgeons or emergency physicians.

Currently, this type of uncertainty is being accepted but it prevents the progressive digitalization of healthcare systems. Value assets should be tagged for protection and also to monitor their functional status. Smart pharmaceuticals are on the horizon but they need to be connected to play their role in individualized medicine scenarios. Automated supply chain monitoring can only work in areas, which are well covered by RATs and patient identification is dependent on wireless communication. The seamless download of digital images to mobile devices for ward rounds and consultations has so far not been an issue as device displays did not deliver on the required resolution for digital medical images. This has changed now and there will be increasing demand for bandwidth on the wards.

\section{BEREC \& NET NEUTRALITY}

The Body of European Regulators for Electronic Communications (BEREC) was established in 2009 by the Regulation (EC) No 1211/2009 of the European Parliament and of the Council. Three strategic pillars of BEREC have been outlined in their strategy 2015-2017 which are of severe relevance for mobile communication, especially in context with the development and implementation of $5 \mathrm{G}$ solutions in health care:

- Promoting competition and investment

- Promoting the internal market

- Empowering and protecting end-users

The most powerful of the pillars is clearly point \#3 "Empowering and protecting end-users" as it is associated with the generation of binding legislation which might pose limitation to stakeholders and market participants during a very sensitive period of implementation of future network technologies, such as $5 \mathrm{G}$ in Europe. Competing interests of stakeholders have already triggered controversial discussions on the consequences of the implementation of RATs and next generation network technologies. A public consultation has been held in order to establish the "BEREC guidelines on the implementation by national regulators of European net neutrality rules. Important and for healthcare infrastructures relevant points are clearly the impact of the nature of healthcare infrastructure as "critical infrastructure" on the net neutrality under circumstances where bandwidth is limited and the demand exceeds the availability and the demarcation of market domains in the provisioning of VPN networks.

\section{PRIVACY AND TRUST IN HEAlthCARE SyStemS}

Privacy is paramount in next generation healthcare infrastructure. Protocols, procedures and architectures of future healthcare networks have to clearly reflect national legislation and European regulation. Public cloud infrastructures, where patient data are shipped to a third party service providers for processing or storage show system immanent weaknesses and are currently not compliant with EC regulations (safe harboring controversy). Also healthcare is considered a "critical infrastructure" meaning it is vital for the functioning of societies and its administrations on a day to day basis. Any dependency on the Internet or remote third party providers could compromise the availability of health care services in case of disaster or exceptional circumstances. Trust in future $5 \mathrm{G}$ solutions is paramount for large scale deployment and commercial exploitation.

Privacy and trust in e-Health focusses on topics such as encrypted message transfer [23], fair non-repudiable message exchange [13], omni-stakeholder fine-grained access control to (Hippocratic) data and the governance of the access policies to such data [16], [24].

\section{SPECTRUM RANGE AND INTEROPERABILITY}

Globally discussions are ongoing about the detailed specifications of $5 \mathrm{G}$ and the different vertical applications and services associated with it. This section deals with core characteristics of $5 \mathrm{G}$ networks as being featured in ongoing discussions.

\section{A. Spectrum}

The different complex, high-end specifications formulated in the context of $5 \mathrm{G}$ will not be achievable within one narrow part of the spectrum. While mobile telephony is typically taking part around 1-2 GHz lower frequencies in the $600-800$ $\mathrm{MHz}$ band are required for Narrowband IoT or similar to allow for better penetration and further reach. Ultra-low latency (less than 5ms) will require far higher frequencies.

Although there are standardization bodies such as 3GPP this does not necessary mean that global frequency band alignment will be a given. There might be frequency allocations in the US ahead of a multilateral agreement. However, following discussions in the European 5G Action Plan Group [25] the following frequencies are under consideration [26]:

- 470-960 MHz band

- $\quad<20 \mathrm{GHz}$ (such as 5.925-6.425GHz, 10-10.68GHz)

- Wave bands between 24 and $43.5 \mathrm{GHz}$

- Wave bands between 66 and $86 \mathrm{GHz}$

During internal discussions within the $5 \mathrm{G}$ Action Plan Group it has been highlighted that "Europe has yet to evaluate the initiatives in the US and Korea regarding the $28 \mathrm{GHz}$ band, bearing in mind the satellite industry is using or planning to use this band and has suggested $31-32 \mathrm{GHz}$ as a better candidate for mobile use (due to global harmonization, similar properties to $28 \mathrm{GHz}$, and also included within WRC-19 studies)" [26].

\section{B. Relevance of Services Contributing to $5 G$ for the Health Care Domain}

$5 \mathrm{G}$ will not only consist of mobile telephony but also include services to integrate smart devices and remote sensors. Examples are Smart Pharmaceuticals such as for example 
asthma inhalers and insulin pens, which will be fitted with narrow band (NB IOT) modules utilizing the $600-900 \mathrm{MHz}$ frequencies for real time provision of virtualized care. These modules might also allow for enhanced mobility and flexibility in hospital settings. NB IOT modules are likely to work under otherwise adverse conditions (in the basement, within steel frame constructions, in old buildings with thick walls, etc.) as the lower frequency spectrum has a better reach and superior penetration potential.

The $28 \mathrm{GHz}$ band has been earmarked by satellite service providers and might be used in healthcare to establish an emergency fallback system in case of natural disaster or other crisis situations where terrestrial services would at least temporarily not be available anymore.

Higher frequencies might play a role in the monitoring of the physical state of car operators in autonomous driving. The envisaged introduction of driverless cars will at least from a regulatory aspect not mean that there is no need for a responsible driver in a car who is in possession of a valid license and also fulfilling all legal requirements to operate a vehicle. It is likely that there will be legislation aiming to ensure that the driver is awake, alert and not intoxicated and in the position to de-activate or override the autonomous driving feature whenever required.

The intended frequency mix is also designed to support new, disruptive services which cannot be foreseen at the moment but which might have a huge commercial impact in the healthcare domain. This could in particular be in context with individualized medicine, smart pharmaceuticals and social care.

\section{Interoperability}

The 5G community needs to work closely with the IOT community to assure end-to-end interoperability with regards to sensors and smart devices in healthcare and ambient assisted living (AAL). Interoperability on this functional level is of high importance not only to prevent fragmentation but also with regards to the implementation of matched $5 \mathrm{G}$ demonstrators with China [27]. In this context the work of the relevant standardization working group of the Association of Internet of Things Innovation (AIOTI) should be considered. Research towards interoperability focusses on areas such as service discovery [28].

\section{CONCLUSION AND OUTLOOK}

This paper provided an overview over the key aspects that will play a role in determining the next generation healthcare network infrastructures. It provided insights into the current state of play in an advanced modern urban hospital in Germany with regards to the utilization of e-Health technology, and outlined the key determinants of future network specifications.

The determination of next generation healthcare network specification is driven by a variety of social and technological factors. Key factors are It is unclear whether all relevant factors have so far been identified. It is, however extremely unlikely that the process of pre-defining requirements in whitepapers or in expert discussions is sufficient to gain a sufficient level of technology readiness [4].

In order to gain confidence with regards to network specifications $5 \mathrm{G}$ demonstrators of different shapes and sizes need to be brought on their way as soon as possible. There can be no doubt that these demonstrators have to start off as $4 \mathrm{G}$ 4.5G LTE solutions and then go through several iterations driven by industrial verticals to progressively evolve step by step. Complexities might be expected through the need for service aggregation and the integration of hardware and software across different networks and domains in a safe and secure manner. However, this can only be successfully managed in time through the involvement of telecom operators, hardware manufacturers and verticals. The European 5G Action Group plan is set to identify concrete projects in Europe until January 2017.

One of the dedicated $5 \mathrm{G}$ health projects will be the Munich based Health 4.0 project at Klinikum rechts der Isar of Technical University Munich, which will build on technical input from Huawei (5G VIA), Nokia (Liquid Apps) and contribution from Siemens and Celestor [29], [30]. In this context the integration of $5 \mathrm{G}$ and IOT technology, Edge Cloud computing and Software to Data and different front end solutions for the health care industry will be tested over a threeyear period starting from January 2017. Mid-term there are plans for a close collaboration with multi-national stakeholders in order to propose detailed specifications for next generation health care networks.

\section{REFERENCES}

[1] R. El Hattachi and J. Erfanian, Eds., 'NGMN 5G Initiative White Paper'. NGMN Alliance, 2015.

[2] 5G PPP, '5G and e-Health'. 2015.

[3] 5g-ppp.eu, ‘5G PPP "5G empowering verticals”@ MWC 2016 in Barcelona'. [Online]. Available: https://5g-ppp.eu/5g-ppp-5gempowering-verticals-mwc-2016-in-barcelona-spain/.

[4] C. Thuemmler, A. K. Lim, I. Holanec, and S. Fricker, 'A methodology to assess social technological alignment in the health domain', IRBM, Jan. 2016.

[5] B. M. C. Silva, J. J. P. C. Rodrigues, I. de la Torre Díez, M. LópezCoronado, and K. Saleem, 'Mobile-health: A review of current state in 2015', Journal of Biomedical Informatics, vol. 56, pp. 265-272, Aug. 2015.

[6] A. Paulin, C. Thuemmler, A. K. Lim, A. Schneider, and H. Feussner, 'eHealth Traffic Analysis and 2035 Future Network Requirements - Part I: State of the Art Service Description', Jan. 2016.

[7] D. Vouyioukas and I. Maglogiannis, 'Pervasive and Smart Technologies for Healthcare: Ubiquitous Methodologies and Tools', in Pervasive and Smart Technologies for Healthcare: Ubiquitous Methodologies and Tools, A. Coronato and G. De Pietro, Eds. IGI Global, 2010, pp. 984 1014.

[8] D. Vouyioukas, I. Maglogiannis, and D. Komnakos, 'Emergency mHealth Services through High-Speed 3G Systems: Simulation and Performance Evaluation', SIMULATION, vol. 83, no. 4, pp. 329-345, Apr. 2007.

[9] D. J. Vergados, D. D. Vergados, and I. Maglogiannis, 'Applying Wireless DiffServ for QoS Provisioning in Mobile Emergency Telemedicine', 2006, pp. 1-5.

[10] Wikipedia, 'BOS - Funk'. [Online]. Available: https://de.wikipedia.org/wiki/BOS-Funk. [Accessed: 10-Dec-2015].

[11] J. Santos, J. J. P. C. Rodrigues, B. M. C. Silva, J. Casal, K. Saleem, and V. Denisov, 'An IoT-based mobile gateway for intelligent personal assistants on mobile health environments', Journal of Network and Computer Applications, vol. 71, pp. 194-204, Aug. 2016. 
[12] A. J. Jara, L. Ladid, and A. Skarmeta, 'The Internet of Everything through IPv6: An Analysis of Challenges, Solutions and Opportunities', Journal of Wireless Mobile Networks, Ubiquitous Computing, and Dependable Applications (JoWUA), vol. 4, no. 3, pp. 97-118, 2013.

[13] A. Paulin and T. Welzer, 'A Universal System for Fair Non-Repudiable Certified e-Mail without a Trusted Third Party', Computers \& Security, 2013.

[14] P. Barnaghi, M. Bauer, A. Rahim Biswas, M. Botterman, B. Cheng, F. Cirillo, M. Dillinger, H. Graux, S. A. Hoseinitabatabaie, E. Kovacs, S. Longo, S. Nunna, A. Paulin, V. Prasad, J. Soldatos, C. Thuemmler, and M. Volk, 'IoT Analytics: Collect, Process, Analyze, and Present Massive Amounts of Operational Data - Research and Innovation Challenges', in Building the hyperconnected society, $\mathrm{O}$. Vermesan and P. Friess, Eds. [S.1.]: River Publishers, 2015.

[15] W. Shi and S. Dustdar, 'The Promise of Edge Computing', Computer, vol. 49 , no. 5 , pp. 78-81, May 2016

[16] A. Paulin, 'Informating Smart Cities Governance? Let Us First Understand the Atoms!', Journal of the Knowledge Economy, Apr. 2016

[17] 'sundhed.dk'. [Online]. Available: https://www.sundhed.dk.

[18] S. Repas, A. Klostermann, C. Seerainer, O. Kuttin, J. Hell, and G. Rauchegger, 'ELGA - Gesamtarchitektur'. ELGA GmbH, 23-Jun-2015.

[19] F. Lupiáñez-Villanueva and A. Theben, 'Title: Strategic Intelligence Monitor on Personal Health Systems Phase 3 (SIMPHS 3) - TDP (United Kingdom) Case Study Report', Institute for Prospective Technological Studies, Joint Research Centre, JRC-IPTS Working Papers JRC94496, 2015.

[20] ISO/IEC, 'ISO/IEC 27001:2013 - Information security management'. 2013.
[21] ISO/IEC, 'ISO/IEC 80001-1:2010'. 2010.

[22] ISO/IEC, 'ISO/IEC 11073-10418:2014'. 2014

[23] B. M. Silva, J. J. Rodrigues, F. Canelo, I. C. Lopes, and L. Zhou, 'A Data Encryption Solution for Mobile Health Apps in Cooperation Environments', Journal of Medical Internet Research, vol. 15, no. 4, p. e66, Apr. 2013.

[24] A. Paulin, 'Towards Self-Service Government - A Study on the Computability of Legal Eligibilities', Journal of Universal Computer Science, vol. 19, no. 12, pp. 1761-1791, Jun. 2013.

[25] European Commission, 'EU and Brazil to work together on 5G mobile technology'. 23-Feb-2016.

[26] 5G Action Group, '5G Action Plan Discussions (unpublished)'. 2016.

[27] P. Friess, X. Yu, P. Cousin, M. Presser, C. Thuemmler, J. Li, X. Zhang, Y. Ge, Y. Liu, H. Li, X. Guan, and C. Bai, 'EU-China White Paper on the Internet of Things'. European Commission, Jan-2016.

[28] P. Desai, A. P. Sheth, and P. Anantharam, 'Semantic Gateway as a Service architecture for IoT Interoperability', CoRR, vol. abs/1410.4977, 2014

[29] Huawei, 'Huawei Builds a Large Scale 5G Testbed in a Real-Life Environment in Munich', Huawei - Media Centre, 26-Feb-2015. [Online]. Available: http://www.huawei.eu/media-centre/pressreleases/huawei-builds-large-scale-5g-testbed-real-life-environmentmunich. [Accessed: 24-Apr-2016].

[30] Nokia, 'Liquid Applications - coming to a cloud near you', 07-Sep2015. [Online]. Available: https://blog.networks.nokia.com/telcocloud/2015/09/07/liquid-applications-coming-to-a-cloud-near-you/. [Accessed: 24-Apr-2016] 